\title{
Investigation of Numerical Solutions of Some Nonlinear Quasiperiodic Differential Equations
}

$\mathrm{By}$

\author{
Taketomo MiTsUI*
}

\section{§ 1. Introduction}

The Galerkin-Urabe's method has had great success in the numerical analysis for periodic solutions of nonlinear periodic differential equations. As an extension of the results, we have tried to deal with nonlinear quasiperiodic oscillations. It is apparently possible to treat many physical phenomena with non-periodic motion as quasiperiodic oscillations. In fact, as far as the author is aware, there are a few papers on the mathematical analysis of quasiperiodic oscillations. In the present paper a numerical method will be proposed and the efficiency of the method will be shown for some nonlinear quasiperiodic differential equations under certain restrictions. In addition, numerical examples will be given in some typical cases.

M. Urabe developed the mathematical theory on the quasiperiodic differential equations ([5], [6], [7]), but did not complete the numeroanalytical methods. On the other hand, some authors have described both the phenomena with quasiperiodic oscillations and the methods for analysis (for instance, [1] and [3]). The present paper, however, may be the first one that contains the numero-analytical methods with precise a posteriori estimates and the numerical examples of the quasiperiodic differential equations.

Our numerical analysis of the quasiperiodic oscillations is based on the following theorem.

Theorem (Urabe [7]). Given a nonlinear differential equation

Received January 28, 1977.

* Research Institute for Mathematical Sciences, Kyoto University, Kyoto 606, Japan. 


$$
\frac{d x}{d t}=X(t, x)
$$

where $x$ and $X(t, x)$ are vectors and $X(t, x)$ is quasiperiodic in $t$ with periods $\omega_{1}, \omega_{2}, \cdots, \omega_{m}$ and is continuously differentiable with respect to $x$ for $x$ belonging to a region $\mathscr{D}$ of the $x$-space.

Suppose that there is a quasiperiodic function $x_{0}(t)$ with periods $\omega_{1}, \omega_{2}, \cdots, \omega_{m}$ such that

$$
\left\{\begin{array}{l}
x_{0}(t) \in \mathscr{D}, \\
\left\|\frac{d x_{0}(t)}{d t}-X\left[t, x_{0}(t)\right]\right\| \leqq r
\end{array}\right.
$$

for all $t$. Further suppose that there are a positive number $\delta$, a non-negative number $\kappa<1$ and a quasiperiodic matrix $A(t)$ with periods $\omega_{1}, \omega_{2}, \cdots, \omega_{m}$ such that

(i) the quasiperiodic differential operator $L$ defined by

$$
L y=\frac{d y}{d t}-A(t) y
$$

is regular as an almost periodic differential operator;

$$
\left\{\begin{array}{l}
\mathscr{D}_{\delta}=\left\{x ;\left\|x-x_{0}(t)\right\| \leqq \delta \text { for some } t\right\} \subset \mathscr{D}, \\
\|\Psi(t, x)-A(t)\| \leqq \frac{\kappa}{M} \text { whenever }\left\|x-x_{0}(t)\right\| \leqq \delta \\
\frac{M r}{1-\kappa} \leqq \delta
\end{array}\right.
$$

Here $\Psi(t, x)$ is the Jacobian matrix of $X(t, x)$ with respect to $x$ and

$$
M=2 C / \sigma \text {, }
$$

where $C$ and $\sigma$ are positive numbers such that Green function $G(t, s)$ for $L$ satisfies

$$
\|G(t, s)\| \leqq C e^{-\sigma|t-s|} .
$$

The given equation (1.1) then posesses a solution $x=\widehat{x}(t)$ quasiperiodic in $t$ with periods $\omega_{1}, \omega_{2}, \cdots, \omega_{m}$ such that

$$
\left\|x_{0}(t)-\widehat{x}(t)\right\| \leqq \frac{M r}{1-\kappa}
$$

for all $t$. For the solution $\hat{x}(t)$, a quasiperiodic differential operator 
$\widehat{L}$ defined by

$$
\widehat{L} y=\frac{d y}{d t}-\Psi[t, \widehat{x}(t)] y
$$

is regular as an almost periodic differential operator. Furthermore, to equation (1.1) there is no other quasiperiodic solution belonging to $\mathscr{D}_{\delta}$ besides $x=\widehat{x}(t)$.

When we apply the above theorem to practical computations we must overcome two difficult points: The one is how to construct an approximate solution $x_{0}(t)$ with enough accuracy satisfying the conditions (1.2), and the other is how to find out a quasiperiodic matrix $A(t)$ which gives a regular quasiperiodic differential operator $L$. Furthermore, for the operator $L$ we must specify the values of constants $C$ and $\sigma$ satisfying (1.6). In order to solve the first point, we shall employ the Galerkin method. To simplify the second point, we shall treat the case in which $X$ in (1.1) is given as a linear term with constant coefficients plus some nonlinear term, and the linear term is taken as the matrix $A(t)$. At the same time, in order to avoid computational complexity we shall consider quasiperiodic systems with only two periods $\omega_{1}$ and $\omega_{2}$, but we believe that this restriction makes no essential change of the problem.

Now we shall give some definitions and propositions on the quasiperiodic differential equations.

A function $f(t)$ is said to be quasiperiodic with periods $\omega_{1}, \omega_{2}, \cdots$, $\omega_{m}$ if $f(t)$ is represented as

$$
f(t)=f_{0}(t, t, \cdots, t)
$$

for some continuous function $f_{0}\left(u_{1}, u_{2}, \cdots, u_{m}\right)$ which is periodic in each $u_{i}$ with period $\omega_{i}$. It is to be noted that a quasiperiodic function is almost periodic. Without loss of generality we may assume that $\omega_{1}, \omega_{2}$, $\cdots, \omega_{m}$ are all positive and further that reciprocals of these periods are rationally linearly independent.

Let $L$ be a differential operator such that

$$
L x=\frac{d x}{d t}-A(t) x
$$


We shall say that $L$ is a quasiperiodic (differential) operator with periods $\omega_{1}, \omega_{2}, \cdots, \omega_{m}$ provided that $A(t)$ is a quasiperiodic matrix with the same periods. The notion of almost periodic (differential) operator is defined analogously.

An almost periodic operator $\mathbb{Q}$ such that

$$
\mathfrak{Q y}=\frac{d y}{d t}-A(t) y
$$

is called to be regular if for any almost periodic function $\psi(t)$ the equation

$$
\mathscr{R} x=\psi(t)
$$

posesses at least one solution bounded for all $t$ ([2]).

Proposition 1 ([2]). \& is regular if and only if there is a square matrix $P$ such that

(i) $P^{2}=P$,

(ii) $\quad\left\|\Phi(t) P \Phi^{-1}(s)\right\| \leqq C e^{-\sigma(t-s)}$ for $t \geqq s$,

(iii) $\quad\left\|\Phi(t)(E-P) \Phi^{-1}(s)\right\| \leqq \mathrm{Ce}^{-\sigma(s-t)}$ for $t<s$,

where $\Phi(t)$ is the fundamental matrix of the linear homogeneous equation

$$
\mathfrak{B}=0
$$

satisfying the initial condition $\Phi(0)=E$ ( $E$ is unit matrix), and $C$ and $\sigma$ are both positive numbers.

The fundamental matrix $\Phi(t)$ satisfying $\Phi(0)=E$ will be called a matrizant hereafter.

A quasiperiodic operator is said to be regular if it is regular as an almost periodic operator.

Proposition 2 ([7]). If a quasiperiodic operator $L$ with periods $\omega_{1}, \omega_{2}, \cdots, \omega_{m}$ defined by (1.10) is regular, then for any quasiperiodic function $f(t)$ with periods $\omega_{1}, \omega_{2}, \cdots, \omega_{m}$ the differential equation 


$$
L x=f(t)
$$

posesses a unique quasiperiodic solution $x=x(t)$ with the same periods and such solution $x(t)$ is given by

$$
x(t)=\int_{-\infty}^{\infty} G(t, s) f(s) d s
$$

where

$$
G(t, s)= \begin{cases}\Phi(t) P \Phi^{-1}(s) & \text { for } t \geq s \\ -\Phi(t)(E-P) \Phi^{-1}(s) & \text { for } t<s .\end{cases}
$$

$G(t, s)$ is called a Green function for $L$, and satisfies the inequality

$$
\|G(t, s)\| \leqq C e^{-\sigma|t-s|}
$$

\section{$\S 2$. Second Order Linear Equation with Constant Coefficients}

In this section we shall analyse a second order linear differential equation with constant coefficients as the preparations for the sections below.

Consider the following quasiperiodic differential equation with periods $\omega_{1}$ and $\omega_{2}$ :

$$
\frac{d^{2} x}{d t^{2}}+2 \mu \frac{d x}{d t}+\nu^{2} x=a \cos \nu_{1} t+b \cos \nu_{2} t
$$

where $\mu, \nu$ are constants such that $\nu>0,0<|\mu|<\nu$, and $\nu_{1}=\frac{2 \pi}{\omega_{1}}, \nu_{2}=\frac{2 \pi}{\omega_{2}}$. From the definition of quasiperiodic function, it is clear that $\omega_{1} / \omega_{2}$ is irrational.

We intend to get quasiperiodic solutions with periods $\omega_{1}$ and $\omega_{2}$ for the equation (1.1). Putting $y=d x / d t$,

$$
\boldsymbol{x}=\left[\begin{array}{l}
x \\
y
\end{array}\right], \quad A=\left[\begin{array}{cc}
0 & 1 \\
-\nu^{2} & -2 \mu
\end{array}\right], \quad \boldsymbol{\varphi}(t)=\left[\begin{array}{c}
0 \\
a \cos \nu_{1} t+b \cos \nu_{2} t
\end{array}\right]
$$

we may rewrite equation (2.1) in the vector form

$$
\frac{d x}{d t}-A x=\varphi(t)
$$

Let $L$ be a differential operator defined by 


$$
L \boldsymbol{x}=\frac{d \boldsymbol{x}}{d t}-A \boldsymbol{x} .
$$

It is easily seen that the matrizant $\Phi(t)$ of the linear homogeneous system

$$
L z=0
$$

is given as follows:

$$
\Phi(t)=\exp t A
$$

$$
=e^{-\mu t}\left(\begin{array}{cc}
\cos \theta t+\frac{\mu}{\theta} \sin \theta t & \frac{1}{\theta} \sin \theta t \\
-\frac{\nu^{3}}{\theta} \sin \theta t & \cos \theta t-\frac{\mu}{\theta} \sin \theta t
\end{array}\right),
$$

where $\theta=\sqrt{\nu^{2}-\mu^{2}}$. We introduce the following $l_{\infty}$-norm of vectors and matrices:

$$
\begin{aligned}
& \|v\|=\max _{i}\left|v_{i}\right| \text { for vectors } v=\left(v_{i}\right), \\
& \|A\|=\max _{i} \sum_{j}\left|a_{i j}\right| \text { for matrices } A=\left(a_{i j}\right) .
\end{aligned}
$$

From (2.5) we then have an estimation to $\Phi(t)$ as follows:

$$
\|\Phi(t)\| \leq C e^{-\mu t}
$$

where

$$
C=\frac{\nu+1}{\theta} \cdot \max (1, \nu) .
$$

Depending on the sign of $\mu$, we have two cases.

Case (I). If $0<\mu<\nu$ holds, we can take $E$ as the matrix $P$ in Proposition 1 , and the Green function for the operator $L$ is given by

$$
G(t, s)=\left\{\begin{array}{cc}
\Phi(t-s) & \text { for } t \geqq s, \\
0 & \text { for } t<s,
\end{array}\right.
$$

$$
=\left\{\begin{array}{lr}
e^{-\mu(t-s)}\left(\begin{array}{lr}
\cos \theta(t-s)+\frac{\mu}{\theta} \sin \theta(t-s) & \frac{1}{\theta} \sin \theta(t-s) \\
-\frac{\nu^{2}}{\theta} \sin \theta(t-s) & \cos \theta(t-s)-\frac{\mu}{\theta} \sin \theta(t-s)
\end{array}\right) \\
0 & \text { for } t \geqq s, \\
0 & \text { for } t<s .
\end{array}\right.
$$


Case (II). If $0>n>-\nu$ holds, we can take 0 as the matrix $P$ in Proposition 1 and the Green function for the operator $L$ is given by

$$
G(t, s)= \begin{cases}0 & \text { for } t \geq s, \\ -\Phi(t-s) & \text { for } t<s,\end{cases}
$$

$$
= \begin{cases}0 & \text { for } t \geqq s, \\
-c^{-\mu(t-s)}\left(\begin{array}{ll}
\cos \theta(t-s)+\frac{\mu}{\theta} \sin \theta(t-s) & \frac{1}{\theta} \sin \theta(t-s) \\
-\frac{\nu^{2}}{\theta} \sin \theta(t-s) & \cos \theta(t-s)-\frac{\mu}{\theta} \sin \theta(t-s)
\end{array}\right) \\
& \text { for } t<s .\end{cases}
$$

In both cases, it is valid that $L$ is regular as the quasiperiodic differential operator with peroids $\omega_{1}$ and $\omega_{2}$. From (1.15), the quasiperiodic solution for equation (2.2) is given by

$$
\boldsymbol{x}(t)=\int_{-\infty}^{t} \Phi(t-s) \varphi(s) d s
$$

for $\mu>0$. Substituting (2.7) into (2.9) and integrating, we have

(2. 10) $x(t)=\frac{a}{\left(\nu^{2}-\nu_{1}^{2}\right)^{2}+4 \mu^{2} \nu_{1}^{2}}\left[\left(\nu^{2}-\nu_{1}^{2}\right) \cos \nu_{1} t+2 \mu \nu_{1} \sin \nu_{1} t\right]$

$$
+\frac{b}{\left(\nu^{2}-\nu_{2}^{2}\right)^{2}+4 \mu^{2} \nu_{2}^{2}}\left[\left(\nu^{2}-\nu_{2}^{2}\right) \cos \nu_{2} t+2 \mu \nu_{2} \sin \nu_{2} t\right] \text {. }
$$

For $\mu<0$, it is easily seen that the result coincides with (2.10). Concluding the considerations, we have

Proposition 3. If $0<|\mu|<\nu$, the operator $L$ defined by (2.3) is regular as the quasiperiodic differential operator with periods $\omega_{1}$ and $\omega_{2}$, and its Green function is given by (2.7) (for $\mu>0$ ) or (2.8) (for $\mu<0$ ). The unique quasiperiodic solution of equation (2.2) (or (2.1)) with periods $\omega_{1}$ and $\omega_{2}$ is given by (2.10).

\section{§3. Duffing Type Equation}

3. 1. In this section, we shall consider a Duffing type equation with quasiperiodic forcing term as the following: 


$$
\frac{d^{2} x}{d t^{2}}+2 \sigma \frac{d x}{d t}+\nu^{2} x=\varepsilon x^{3}+a \cos \nu_{1} t+b \cos \nu_{2} t
$$

where $0<\sigma<\nu, \varepsilon$ is a positive parameter, $\nu_{1}=\frac{2 \pi}{\omega_{1}}, \nu_{2}=\frac{2 \pi}{\omega_{2}}$ and $\omega_{2} / \omega_{1}$ is irrational.

Let us write the equation (3.1) in the form

$$
\frac{d \boldsymbol{x}}{d t}=A \boldsymbol{x}+\boldsymbol{\varphi}(t)+\varepsilon \boldsymbol{\zeta}(\boldsymbol{x})
$$

where

$$
\begin{aligned}
& \boldsymbol{x}=\left[\begin{array}{l}
x \\
y
\end{array}\right], \quad A=\left[\begin{array}{cc}
0 & 1 \\
-\nu^{2} & -2 \sigma
\end{array}\right], \quad \boldsymbol{\varphi}(t)=\left[\begin{array}{c}
0 \\
a \cos \nu_{1} t+b \cos \nu_{2} t
\end{array}\right], \\
& \boldsymbol{\zeta}(\boldsymbol{x})=\left[\begin{array}{l}
0 \\
x^{3}
\end{array}\right] .
\end{aligned}
$$

Let $L$ be a differential operator defined by

$$
L z=\frac{d z}{d t}-A z
$$

then by Proposition 3 we see that $L$ is regular as a quasiperiodic differential operator, the Green function for $L$ satisfies

$$
\|G(t, s)\| \leqq C e^{-\sigma|t-s|},
$$

where $C=\frac{\nu+1}{\sqrt{\nu^{2}-\sigma^{2}}} \cdot \max (1, \nu)$, and the quasiperiodic solution of the equation

$$
L \boldsymbol{x}=\boldsymbol{\varphi}(t)
$$

is given by $\boldsymbol{x}=\boldsymbol{x}_{0}(t)=\left[\begin{array}{l}x_{0}(t) \\ y_{0}(t)\end{array}\right]$,

where

$$
\begin{aligned}
x_{0}(t)= & \frac{a}{\left(\nu^{2}-\nu_{1}^{2}\right)^{2}+4 \sigma^{2} \nu_{1}^{2}}\left[\left(\nu^{2}-\nu_{1}^{2}\right) \cos \nu_{1} t+2 \sigma \nu_{1} \sin \nu_{1} t\right] \\
& +\frac{b}{\left(\nu^{2}-\nu_{2}^{2}\right)^{2}+4 \sigma^{2} \nu_{2}^{2}}\left[\left(\nu^{2}-\nu_{2}^{2}\right) \cos \nu_{2} t+2 \sigma \nu_{2} \sin \nu_{2} t\right], \\
y_{0}(t)= & \frac{d}{d t} x_{0}(t) .
\end{aligned}
$$


For sufficiently small $\varepsilon, \boldsymbol{x}_{0}(t)$ may be regarded as an approximate solution to the equation (3.1). In fact, it is shown in [7] that for sufficiently small $\varepsilon$ the equation (3.1) possesses a quasiperiodic solution $\boldsymbol{x}=\widehat{x}(t)$ with periods $\omega_{1}$ and $\omega_{2}$ in some neighbourhood of $x_{0}(t)$.

\section{2. Galerkin Scheme}

From the definition of the quasiperiodic function, there exists a continuous multiperiodic function which corresponds to a quasiperiodic function. Considering this fact, we introduce a partial differential system which is doubly periodic:

$$
D \tilde{\boldsymbol{x}}=A \tilde{\boldsymbol{x}}+\hat{\boldsymbol{\varphi}}\left(u_{1}, u_{2}\right)+\varepsilon \hat{\boldsymbol{\zeta}}(\tilde{\boldsymbol{x}}),
$$

where

$$
\begin{aligned}
& \tilde{\boldsymbol{x}}=\left[\begin{array}{l}
\tilde{\boldsymbol{x}}\left(u_{1}, u_{2}\right) \\
\widetilde{\boldsymbol{y}}\left(u_{1}, u_{2}\right)
\end{array}\right], \quad D=\frac{\partial}{\partial u_{1}}+\frac{\partial}{\partial u_{2}}, \\
& A=\left[\begin{array}{cc}
0 & 1 \\
-\nu^{2} & -2 \sigma
\end{array}\right], \quad \tilde{\boldsymbol{\varphi}}\left(u_{1}, u_{2}\right)=\left[\begin{array}{c}
0 \\
a \cos \nu_{1} u_{1}+b \cos \nu_{2} u_{2}
\end{array}\right], \\
& \tilde{\boldsymbol{\zeta}}(\boldsymbol{x})=\left[\begin{array}{c}
0 \\
\tilde{x}^{3}
\end{array}\right] .
\end{aligned}
$$

It is evident that if $\widetilde{\boldsymbol{x}}=\widetilde{\boldsymbol{x}}\left(u_{1}, u_{2}\right)$ is a doubly periodic solution with periods $\omega_{1}$ and $\omega_{2}$ of the equation (3.7), then $\boldsymbol{x}(t)=\tilde{\boldsymbol{x}}(t, t)$ is a quasiperiodic solution of (3.2). We shall solve approximately the equation (3.7) by the Galerkin method.

Using the doubly periodic finite Fourier basis with periods $\omega_{1}$ and $\omega_{2}$, we approximate the doubly periodic solutions by

$$
\left\{\begin{array}{l}
x_{m}\left(u_{1}, u_{2}\right)=\alpha(0,0)+\sum_{r=1}^{m} \sum_{|p|=r}\left\{\alpha_{p} \cos (p, \nu, u)+\beta_{p} \sin (p, \nu, u)\right\} \\
y_{m}\left(u_{1}, u_{2}\right)=\alpha^{\prime}(0,0)+\sum_{r=1}^{m} \sum_{|p|=r}\left\{\alpha_{p}^{\prime} \cos (p, \nu, u)+\beta_{p}^{\prime} \sin (p, \nu, u)\right\}
\end{array}\right.
$$

where $p$ is a pair of integers $p=\left(p_{1}, p_{2}\right), \boldsymbol{u}={ }^{t}\left(u_{1}, u_{2}\right)$,

$$
\begin{aligned}
& (p, \nu, \boldsymbol{u})=p_{1} \nu_{1} u_{1}+p_{2} \nu_{2} u_{2}, \\
& |p|=\left|p_{1}\right|+\left|p_{2}\right|,
\end{aligned}
$$


and the symbol $\sum_{|p|=r}$ signifies the summation for all $p$ such that $|p|=r$. If the function $x_{m}\left(u_{1}, u_{2}\right)$ is given, then it is clear that

$$
\left\{\begin{array}{l}
\alpha_{p}=\frac{2}{\omega_{1} \omega_{2}} \int_{0}^{\omega_{1}} \int_{0}^{\omega_{2}} x_{m}(\boldsymbol{u}) \cos (p, \nu, \boldsymbol{u}) d u_{1} d u_{2}, \\
\beta_{p}=\frac{2}{\omega_{1} \omega_{2}} \int_{0}^{\omega_{1}} \int_{0}^{\omega_{2}} x_{m}(u) \sin (p, \nu, \boldsymbol{u}) d u_{1} d u_{2}, \\
\alpha(0,0)=\frac{1}{\omega_{1} \omega_{2}} \int_{0}^{\omega_{1}} \int_{0}^{\omega_{2}} x_{m}(\boldsymbol{u}) d u_{1} d u_{2} .
\end{array}\right.
$$

It should be remarked that the coefficients $\alpha_{p} \equiv \alpha\left(p_{1}, p_{2}\right)$ and $\beta_{p} \equiv \beta\left(p_{1}, p_{2}\right)$ have the following property

$$
\alpha_{-p}=\alpha_{p}, \beta_{-p}=-\beta_{p} \quad\left(-p=\left(-p_{1},-p_{2}\right)\right)
$$

because of the symmetricity of the cosine function and the anti-symmetricity of the sine function (the same as for $\alpha_{p}{ }^{\prime}$ and $\beta_{p}{ }^{\prime}$ ).

On the location of pairs of integers $p=\left(p_{1}, p_{2}\right)$, we adopt the following order:

(i) The pairs are arranged in the ascending order of their absolute values, i.e. $|p|=0,1,2, \cdots$.

(ii) Among the pairs $\left(p_{1}, p_{2}\right)$ with the same absolute value $m$, we shall arrange them in the descending order of the absolute value of $p_{1}$, i.e. $\left|p_{1}\right|=m, m-1, \cdots, 1,0$.

(iii) If $p_{1} \neq 0$ and $p_{2} \neq 0$, the four pairs $\left(p_{1}, p_{2}\right)$ with $\left|p_{1}\right|+\left|p_{2}\right|=m$ and $\left|p_{1}\right|=l$ are arranged as follows: $(l, m-l),(-l,-(m-l)),(l,-(m$ $-l)),(-l, m-l)$.

(iv) If $p_{2}=0$ and $|p|=m$, the order is $(m, 0),(-m, 0)$. If $p_{1}=0$ and $|p|=m$, the order is $(0, m),(0,-m)$.

Hereafter the following notation will be used.

$$
\mathcal{I}\{f(\boldsymbol{u})\}=\frac{1}{\omega_{1} \omega_{2}} \int_{0}^{\omega_{1}} \int_{0}^{\omega_{2}} f\left(u_{1}, u_{2}\right) d u_{1} d u_{2} .
$$

The unknown coefficients $\alpha_{p}, \beta_{p}, \alpha_{p}{ }^{\prime}$ and $\beta_{p}{ }^{\prime}$ are determined in order that $D \boldsymbol{x}_{m}-A \boldsymbol{x}_{m}-\tilde{\boldsymbol{\varphi}}(\boldsymbol{u})-\varepsilon \tilde{\boldsymbol{\zeta}}\left(\boldsymbol{x}_{m}\right)$ may be orthogonal to the doubly periodic finite Fourier basis with $|p| \leqq m$. From the equations

$$
\left\{\begin{array}{l}
2 \mathcal{I}\left[\left\{D x_{m}(\boldsymbol{u})-y_{m}(\boldsymbol{u})\right\}\left\{\begin{array}{l}
\cos (p, \nu, \boldsymbol{u}) \\
\sin (p, \nu, \boldsymbol{u})
\end{array}\right\}\right]=0, \\
\mathcal{G}\left[D x_{m}(\boldsymbol{u})-y_{m}(\boldsymbol{u})\right]=0
\end{array}\right.
$$


we obtain

$$
\left\{\begin{array}{l}
\alpha^{\prime}(0,0)=0, \\
\alpha_{p}^{\prime}=\left(p_{1} \nu_{1}+p_{2} \nu_{2}\right) \beta_{p}, \\
\beta_{p}^{\prime}=-\left(p_{1} \nu_{1}+p_{2} \nu_{2}\right) \alpha_{p} .
\end{array}\right.
$$

Substitution of the relations (3.13) in the second equality of (3.8) yields the following determining equations:

$(3.14)_{1} \quad f_{(0,0)}^{(m)}\left(\alpha^{(m)}\right) \equiv \nu^{2} \alpha(0,0)-\varepsilon \mathcal{G}\left\{x_{m}{ }^{3}(\boldsymbol{u})\right\}=0$,

(3. 14) $)_{2} \quad f_{(1,0)}^{(m)}\left(\alpha^{(m)}\right) \equiv\left(\nu^{2}-\nu_{1}^{2}\right) \alpha(1,0)+2 \sigma \nu_{1} \beta(1,0)-\frac{a}{2}$

$-2 \varepsilon \mathcal{G}\left\{x_{m}{ }^{3}(\boldsymbol{u}) \cos \nu_{1} u_{1}\right\}=0$,

$(3.14)_{3} \quad f_{(0,1)}^{(m)}\left(\alpha^{(m)}\right) \equiv\left(\nu^{2}-\nu_{2}^{2}\right) \alpha(0,1)+20 \nu_{2} \beta(0,1)-\frac{b}{2}$

$$
-2 \mathcal{E} \mathcal{G}\left\{x_{m}{ }^{3}(\boldsymbol{u}) \cos \nu_{2} u_{2}\right\}=0,
$$

$(3.14)_{4} \quad f_{\left(p_{1}, p_{2}\right)}^{(m)}\left(\alpha^{(m)}\right) \equiv\left\{\nu^{2}-\left(p_{1} \nu_{1}+p_{2} \nu_{2}\right)^{2}\right\} \alpha_{p}+2 \sigma\left(p_{1} \nu_{1}+p_{2} \nu_{2}\right) \beta_{p}$

$$
-2 \varepsilon \mathcal{G}\left\{x_{m}{ }^{3}(\boldsymbol{u}) \cos (p, \nu, u)\right\}=0 \quad \text { for }|p| \geqq 2,
$$

$(3.14)_{5} \quad g_{\left(p_{1}, p_{2}\right)}^{(m)}\left(\alpha^{(m)}\right) \equiv\left\{\nu^{2}-\left(p_{1} \nu_{1}+p_{2} \nu_{2}\right)^{2}\right\} \beta_{p}-2 \sigma\left(p_{1} \nu_{1}+p_{2} \nu_{2}\right) \alpha_{p}$

$$
-2 \varepsilon \mathcal{G}\left\{x_{m}{ }^{3}(\boldsymbol{u}) \sin (p, \boldsymbol{\nu}, \boldsymbol{u})\right\}=0 \text { for }|p| \geqq 1 \text {. }
$$

For brevity, let us denote the determining equations (3. 14) $\sim(3.14)_{5}$ by $F_{m}\left(\alpha^{(m)}\right)=0$, where $\alpha^{(m)}={ }^{t}\left(\alpha(0,0), \cdots, \alpha_{p}, \beta_{p}, \cdots\right), \mid p_{1} \leqq m, p_{1} \geqq 0$. $F_{m}\left(\alpha^{(m)}\right)=0$ is a system of $2 m(m+1)+1$ equations for $2 m(m+1)+1$ unknowns.

For the elements of $\alpha^{(m)}$, considering the property (3.10), we adopt the location order similar to that for the pairs $\left(p_{1}, p_{2}\right)$. The rules (iii) and (iv) are, however, replaced with the following.

(iii)' If $p_{1} \neq 0$ and $p_{2} \neq 0, \alpha\left(p_{1}, p_{2}\right)$ and $\beta\left(p_{1}, p_{2}\right)$ with $\left|p_{1}\right|+\left|p_{2}\right|=m$ and $\left|p_{1}\right|=l$ are arranged as follows: $\alpha(l, m-l), \beta(l, m-l), \alpha(l,-(m-l))$, $\beta(l,-(m-l))$.

(iv)' If $p_{2}=0$ and $|p|=m$, the order is $\alpha(m, 0), \beta(m, 0)$.

If $p_{1}=0$ and $|p|=m$, the order is $\alpha(0, \mathrm{~m}), \beta(0, m)$.

\section{3. An Iterative Process}

The determining equation $F_{m}\left(\alpha^{(m)}\right)=0$ is equivalent to the equation 


$$
A_{m} \alpha^{(m)}-\boldsymbol{a}=\varepsilon C_{m}\left(\alpha^{(m)}\right),
$$

where

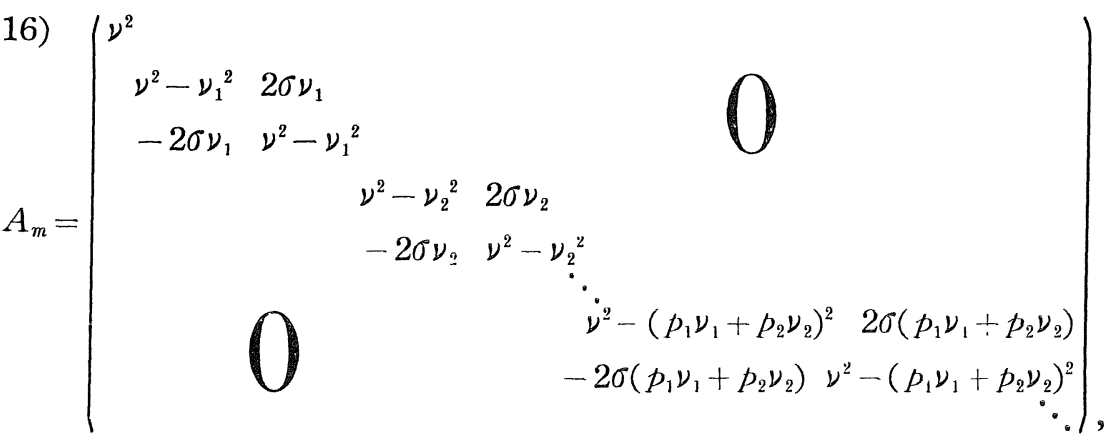

$$
\boldsymbol{a}={ }^{t}(0, a / 2,0, b / 2,0, \cdots, 0),
$$

and $C_{m}\left(\alpha^{(m)}\right)$ is the nonlinear function of $\alpha^{(m)}$ such that

$$
\begin{aligned}
& \text { (3. 18) } C_{m}\left(\alpha^{(m)}\right)={ }^{t}\left(c_{0}, \cdots, c_{p}, d_{p}, \cdots\right)_{|p| \leqq m, p_{1} \geq 0}, \\
& \text { (3. 19) } c_{p}=2 \mathcal{G}\left\{x_{m}{ }^{3}(\boldsymbol{u}) \cos (p, \nu, \boldsymbol{u})\right\}, d_{\rho}=2 \mathcal{I}\left\{x_{m}{ }^{3}(\boldsymbol{u}) \sin (p, \boldsymbol{\nu}, \boldsymbol{u})\right\} \\
& c_{0}=\mathcal{I}\left\{x_{m}{ }^{3}(\boldsymbol{u})\right\} .
\end{aligned}
$$

Since $A_{m}$ is a block diagonal matrix, it is easily seen that

$$
\text { (3. 20) } \operatorname{det} A_{m}=\nu^{2} \prod_{\substack{1 \leq p_{1} \leq m \\ p_{1} \geqq 0}}\left[\left\{\nu^{2}-\left(p_{1} \nu_{1}+p_{2} \nu_{2}\right)^{2}\right\}^{2}+4 \sigma^{2}\left(p_{1} \nu_{1}+p_{2} \nu_{2}\right)^{2}\right] \text {. }
$$

The inequality

$$
\left\{\nu^{2}-\left(p_{1} \nu_{1}+p_{2} \nu_{2}\right)^{2}\right\}^{2}+4 \sigma^{2}\left(p_{1} \nu_{1}+p_{2} \nu_{2}\right)^{2} \geqq 4 \sigma^{2}\left(\nu^{2}-\sigma^{2}\right)
$$

holds for all $\left(p_{1}, p_{2}\right)$. Thus $A_{m}$ is a nonsingular matrix under the assumption $0<\sigma<\nu$.

Considering that $\varepsilon$ is a small parameter, we may define an iterative process as follows:

Starting from a suitable initial value $\alpha_{0}{ }^{(m)}$, we make a sequence of vectors $\left\{\alpha_{k}^{(m)}\right\}$ such that

$$
\alpha_{k}^{(m)}=\varepsilon A_{m}{ }^{-1} C_{m}\left(\alpha_{k-1}^{(m)}\right)+A_{m}{ }^{-1} \boldsymbol{\alpha}, \quad k=1,2, \cdots .
$$

Assume that there exists a solution $\hat{\alpha}^{(m)}$ of the equation $F_{m}\left(\alpha^{(m)}\right)=0$. Because of the formulas (3.8) and (3.19), $C_{m}\left(\alpha^{(m)}\right)$ continuously depends on $\alpha^{(m)}$. Hence, if we can take sufficiently small $\varepsilon$ and the initial value $\alpha_{0}{ }^{(m)}$ which belongs to sufficiently small neighbourhood of $\hat{\alpha}^{(m)}$, the iter- 
ative process (3.22) would be expected to be convergent to $\hat{\alpha}^{(m)}$.

\section{4. A posteriori Estimates}

After some iterations we have an approximate solution $\bar{\alpha}^{(m)}$ of the determining equation $F_{m}\left(\alpha^{(m)}\right)=0$, which means that to the equation (3.1) we have an approximate solution $\bar{x}_{m}(t)$ such that

$$
\bar{x}_{m}(t)=\bar{\alpha}(0,0)+\sum_{r=1}^{m} \sum_{|p|=r}\left\{\bar{\alpha}_{p} \cos (p, \nu) t+\bar{\beta}_{p} \sin (p, \nu) t\right\}
$$

where $(p, \nu)=p_{1} \nu_{1}+p_{2} \nu_{2}$. After having found the approximate solution $\bar{x}_{m}(t)$, it is necessary to verify the existence of the exact solution and to give a posteriori error estimate for the approximate solution $\bar{x}_{m}(t)$.

Let us define the residual function to $\bar{x}_{m}(t)$ as follows:

$$
r(t)=\frac{d^{2} \bar{x}_{m}(t)}{d t^{2}}+2 \sigma \frac{d \bar{x}_{m}(t)}{d t}+\nu^{2} \bar{x}_{m}(t)-\varepsilon \bar{x}_{m}{ }^{3}(t)-a \cos \nu_{1} t-b \cos \nu_{2} t
$$

The coefficients of the double Fourier series expansion of $r(t)$ are just given by the elements of the residual of determining equation to $\bar{\alpha}^{(m)}$. In the present case, since the nonlinear term of the right-hand side of (3. 24) is of the third power of $\bar{x}_{m}(t)$, the double Fourier series of $r(t)$ is finite, so that we have the equality such that

$$
r(t)=f(0,0)+\sum_{r=1}^{3 m} \sum_{|p|=r}\left\{f_{p} \cos (p, \nu) t+g_{p} \sin (p, \nu) t\right\}
$$

where the coefficients $f_{p}$ and $g_{p}$ satisfy the following relations with $f_{p}^{(m)}\left(\alpha^{(m)}\right)$ and $g_{p}{ }^{(m)}\left(\alpha^{(m)}\right)$ in (3.14):

$$
\begin{cases}f_{(0,0)} & =f_{(0,0)}^{(m)}\left(\bar{\alpha}^{(m)}\right), \\ f_{p}= \begin{cases}f_{p}{ }^{(m)}\left(\bar{\alpha}^{(m)}\right) & \text { for }|p| \leqq m, \\ -2 \varepsilon \mathcal{G}\left\{\bar{x}_{m}{ }^{3}(\boldsymbol{u}) \cos (p, \boldsymbol{\nu}, \boldsymbol{u})\right\} & \text { for }|p|>m,\end{cases} \\ g_{p}= \begin{cases}g_{p}^{(m)}\left(\bar{\alpha}^{(m)}\right) & \text { for }|p| \leqq m, \\ -2 \varepsilon \mathcal{J}\left\{\bar{x}_{m}{ }^{3}(\boldsymbol{u}) \sin (p, \boldsymbol{\nu}, \boldsymbol{u})\right\} & \text { for }|p|>m .\end{cases} \end{cases}
$$

Consequently we can take such an $r$ in (1.2) that

$$
r \geqq\left|f_{(0,0)}\right|+\sum_{r=1}^{3 m} \sum_{|p|=r}\left(\left|f_{p}\right|+\left|g_{p}\right|\right) \text {. }
$$


Next, we denote $\omega$ by

$$
\omega=|\bar{\alpha}(0,0)|+\sum_{r=1}^{m} \sum_{|p|=r}\left(\left|\bar{\alpha}_{p}\right|+\beta_{p} \mid\right),
$$

then the inequality

$$
\omega \geqq \sup \left|\bar{x}_{m}(t)\right|
$$

holds. For the equation (3.2), the Jacobian matrix with respect to $\boldsymbol{x}$ is

$$
\Psi(t, \boldsymbol{x})=\left[\begin{array}{cc}
0 & 1 \\
-\nu^{2}+3 \varepsilon x^{2} & -2 \sigma
\end{array}\right] .
$$

Hence we have

$$
\|\Psi(t, \boldsymbol{x})-A\|=\left|3 \varepsilon x^{2}\right|=3 \varepsilon|x|^{2} .
$$

For $x$ which is in the $\delta$-neighbourhood of $\bar{x}_{m}(t)$, the inequality

$$
|x| \leqq\left|\bar{x}_{m}(t)\right|+\delta
$$

holds. Therefore we obtain from (3.29), (3.30) and (3.31) that

$$
\|\Psi(t, \boldsymbol{x})-A\| \leqq 3 \varepsilon(\omega+\delta)^{2} .
$$

By (1.5), (1.6) and (3.4), we get

$$
M=\frac{2 \nu(1+\nu)}{\sigma \sqrt{\nu^{2}-\sigma^{2}}}
$$

under the assumption $\nu \geqq 1$.

If there exist a non-negative number $\kappa<1$ and a positive number $\delta$ satisfying the both of inequalities

$$
\begin{gathered}
3 \varepsilon(\omega+\delta)^{2} \leqq \frac{\kappa \sigma \sqrt{\nu^{2}-\sigma^{2}}}{2 \nu(1+\nu)}, \\
\frac{r}{1-\kappa} \cdot \frac{2 \nu(1+\nu)}{\sigma \sqrt{\nu^{2}-\sigma^{2}}} \leqq \delta,
\end{gathered}
$$

the condition (1.4) of the Theorem is fulfilled. Hence the exact quasiperiodic solution $\widehat{x}(t)$ with periods $\omega_{1}$ and $\omega_{2}$ exists and an error estimation of $\bar{x}_{m}(t)$ is given by

$$
\left|\bar{x}_{m}(t)-\widehat{x}(t)\right| \leqq \frac{r}{1-\kappa} \frac{2 \nu(1+\nu)}{\sigma \sqrt{\nu^{2}-\sigma^{2}}}
$$

for all $t$ 


\section{$\S$ 4. Van der Pol Type Equation}

4.1. In this section, we shall consider a van der Pol type equation with a quasiperiodic forcing term as following:

$$
\frac{d^{2} x}{d t^{2}}-2 \lambda\left(1-x^{2}\right) \frac{d x}{d t}+x=a \cos \nu_{1} t+b \cos \nu_{2} t,
$$

where $\lambda$ is a positive parameter, $\nu_{1}=2 \pi / \omega_{1}, \nu_{2}=2 \pi / \omega_{2}, \omega_{2} / \omega_{1}$ is irrational, and neither $\nu_{1}$ nor $\nu_{2}$ is equal to 1 .

Let us rewrite the equation (4.1) in the form

$$
\frac{d \boldsymbol{x}}{d t}=A(\lambda) \boldsymbol{x}+\boldsymbol{\varphi}(t)+\lambda \boldsymbol{\eta}(\boldsymbol{x})
$$

where

$$
\begin{aligned}
& \boldsymbol{x}=\left[\begin{array}{l}
x \\
y
\end{array}\right], \quad A(\lambda)=\left[\begin{array}{cc}
0 & 1 \\
-1 & 2 \lambda
\end{array}\right], \quad \boldsymbol{\varphi}(t)=\left[\begin{array}{c}
0 \\
a \cos \nu_{1} t+b \cos \nu_{2} t
\end{array}\right], \\
& \boldsymbol{\eta}(\boldsymbol{x})=\left[\begin{array}{c}
0 \\
-2 x^{2} y
\end{array}\right]
\end{aligned}
$$

Let $L(\lambda)$ be a differential operator depending on $\lambda$ such that

$$
L(\lambda) z=\frac{d z}{d t}-A(\lambda) z .
$$

We apply Proposition 3 to the case $\mu=-\lambda \nu=1$. Then we have that $L(\lambda)$ is regular as a quasiperiodic operator for $\lambda<1$ and that the Green function for $L(\lambda)$ is given by

$$
G_{\lambda}(t, s)= \begin{cases}0 & \text { for } t \geq s, \\
-e^{\lambda(t-s)}\left(\begin{array}{ll}
\cos \theta(t-s)-\frac{\lambda}{\theta} \sin \theta(t-s) & \frac{1}{\theta} \sin \theta(t-s) \\
-\frac{1}{\theta} \sin \theta(t-s) & \cos \theta(t-s)+\frac{\lambda}{\theta} \sin \theta(t-s)
\end{array}\right) & \text { for } t<s,\end{cases}
$$

where $\theta=\sqrt{1-\lambda^{2}}$. Therefore, the Green function $G_{\lambda}(t, s)$ satisfies

$$
\left\|G_{\lambda}(t, s)\right\| \leqq \frac{2-\lambda^{2}}{\theta} e^{-\lambda|t-s|},
$$

and the quasiperiodic solution of the linear equation

$$
L(\lambda) \boldsymbol{w}=\boldsymbol{\varphi}(t)
$$


is given by $\boldsymbol{w}=\boldsymbol{w}_{0}(t ; \lambda)=\left[\begin{array}{c}x_{0}(t ; \lambda) \\ y_{0}(t ; \lambda)\end{array}\right]$, where

(4. 7)

$$
\left\{\begin{aligned}
x_{0}(t ; \lambda)= & \frac{a}{\left(1-\nu_{1}^{2}\right)^{2}+4 \lambda^{2} \nu_{1}^{2}}\left\{\left(1-\nu_{1}^{2}\right) \cos \nu_{1} t-2 \lambda \nu_{1} \sin \nu_{1} t\right\} \\
& +\frac{b}{\left(1-\nu_{2}^{2}\right)^{2}+4 \lambda^{2} \nu_{2}^{2}}\left\{\left(1-\nu_{2}^{2}\right) \cos \nu_{2} t-2 \lambda \nu_{2} \sin \nu_{2} t\right\}, \\
y_{0}(t ; \lambda)= & \frac{a \nu_{1}}{\left(1-\nu_{1}^{2}\right)^{2}+4 \lambda^{2} \nu_{1}^{2}}\left\{-\left(1-\nu_{1}^{2}\right) \sin \nu_{1} t-2 \lambda \nu_{1} \cos \nu_{1} t\right\} \\
& +\frac{b \nu_{2}}{\left(1-\nu_{2}^{2}\right)^{2}+4 \lambda^{2} \nu_{2}^{2}}\left\{-\left(1-\nu_{2}^{2}\right) \sin \nu_{2} t-2 \lambda \nu_{2} \cos \nu_{2} t\right\} .
\end{aligned}\right.
$$

4. 2. Define the constant $K$ by

$$
K=\max \left(\frac{|a|}{\left|1-\nu_{1}^{2}\right|}+\frac{|b|}{\left|1-\nu_{2}^{2}\right|}, \frac{|a| \nu_{1}}{\left|1-\nu_{1}^{2}\right|}+\frac{|b| \nu_{2}}{\left|1-\nu_{2}^{2}\right|}\right) .
$$

We shall show that under the assumption $K<\sqrt{\frac{\sqrt{1-\lambda^{2}}}{52\left(2-\lambda^{2}\right)}}$ there exists a quasiperiodic solution with periods $\omega_{1}$ and $\omega_{2}$ to the nonlinear equation (4. 2) in a neighbourhood of $\boldsymbol{w}_{0}(t ; \lambda)$. Let us remark that $K$ does not depend on $\lambda$. From the formula (4.7), we have

$$
x_{0}(t ; \lambda)=\frac{a \sin \left(\nu_{1} t+\alpha\right)}{\left\{\left(1-\nu_{1}^{2}\right)^{2}+4 \lambda^{2} \nu_{1}^{2}\right\}^{1 / 2}}+\frac{b \sin \left(\nu_{2} t+\beta\right)}{\left\{\left(1-\nu_{2}^{2}\right)^{2}+4 \lambda^{2} \nu_{2}^{2}\right\}^{1 / 2}},
$$

where $\alpha$ and $\beta$ satisfy

$$
\tan \alpha=-\frac{1-\nu_{1}{ }^{2}}{2 \lambda \nu_{1}}, \quad \tan \beta=-\frac{1-\nu_{2}^{2}}{2 \lambda \nu_{2}} .
$$

Then, by differentiation we have

$$
\text { (4. 10) } y_{0}(t ; \lambda)=\frac{a \nu_{1} \cos \left(\nu_{1} t+\alpha\right)}{\left\{\left(1-\nu_{1}^{2}\right)^{2}+4 \lambda^{2} \nu_{1}^{2}\right\}^{1 / 2}}+\frac{b \nu_{2} \cos \left(\nu_{2} t+\beta\right)}{\left\{\left(1-\nu_{2}^{2}\right)^{2}+4 \lambda^{2} \nu_{2}^{2}\right\}^{1 / 2}} \text {. }
$$

For all $t$ we obtain

$$
\begin{aligned}
\left|x_{0}(t ; \lambda)\right| & \leqq \frac{|a|}{\left\{\left(1-\nu_{1}^{2}\right)^{2}+4 \lambda^{2} \nu_{1}{ }^{2}\right\}^{1 / 2}}+\frac{|b|}{\left\{\left(1-\nu_{2}^{2}\right)^{2}+4 \lambda^{2} \nu_{2}\right\}^{1 / 2}} \\
& \leqq \frac{|a|}{\left|1-\nu_{1}{ }^{2}\right|}+\frac{|b|}{\left|1-\nu_{2}{ }^{2}\right|},
\end{aligned}
$$




$$
\left|y_{0}(t ; \lambda)\right| \leqq \frac{|a| \nu_{1}}{\left|1-\nu_{1}^{2}\right|}+\frac{|b| \nu_{2}}{\left|1-\nu_{2}^{2}\right|}
$$

Therefore we get

$$
\left|x_{0}(t ; \lambda)\right|, \quad\left|y_{0}(t ; \lambda)\right|<K
$$

for all $t$ and $0<\lambda<1$. We can give the residual erior of the equation (4. 2) for $\boldsymbol{w}_{0}(t ; \lambda)$ in the following form:

$$
\begin{aligned}
& \left\|\frac{d \boldsymbol{w}_{0}(t ; \lambda)}{d t}-A(\lambda) \boldsymbol{w}_{0}(t ; \lambda)-\boldsymbol{\varphi}(t)-\lambda \boldsymbol{\eta}\left(\boldsymbol{w}_{0}(t ; \lambda)\right)\right\| \\
& \quad=\left\|-\lambda \boldsymbol{\eta}\left(\boldsymbol{w}_{0}(t ; \lambda)\right)\right\| \\
& \quad \leqq 2 \lambda\left|x_{0}{ }^{2}(t ; \lambda) y_{0}(t ; \lambda)\right| \\
& \quad \leqq 2 \lambda K^{3} .
\end{aligned}
$$

Hence we may set

$$
r=2 \lambda K^{3}
$$

Let $\mathscr{D}_{K}$ and $\mathscr{D}^{\prime}$ be the domains in $\boldsymbol{R}^{2}$ such that

$$
\begin{aligned}
& \mathscr{D}_{K}=\{x ;\|x\| \leqq 2 K\}, \\
& \mathscr{D}^{\prime}=\bigcup_{\iota}\left\{\boldsymbol{x} ;\left\|\boldsymbol{x}-\boldsymbol{w}_{0}(t ; \lambda)\right\| \leqq K\right\} .
\end{aligned}
$$

It is clear that $\boldsymbol{w}_{0}(t ; \lambda) \in \mathscr{D}_{K}$ for any $t$ and $\mathscr{D}^{\prime} \subset \mathscr{D}_{K}$.

Let us denote the Jacobian matrix of the right-hand side of (4.2) with respect to $\boldsymbol{x}$ by $\Psi(\boldsymbol{x} ; \lambda)$. Then we have

$$
\|\Psi(x ; \lambda)-A(\lambda)\| \leqq 2 \lambda(2|y|+|x|)|x| \leqq 24 \lambda K^{2}
$$

for $\boldsymbol{x} \in \mathscr{D}^{\prime}$. The inequality (4.5) yields

$$
M=\frac{2\left(2-\lambda^{2}\right)}{\lambda \sqrt{ } 1-\lambda^{2}}
$$

In order to apply the Theorem to the present case, we have to check with the inequalities in (1.4). The question is "Is it possible to take a nonnegative number $\kappa<1$ satisfying the both of inequalities

$$
24 \lambda K^{2} \leqq \frac{\lambda \sqrt{1-\lambda^{2}}}{2\left(2-\lambda^{2}\right)} \kappa
$$




$$
\frac{2\left(2-\lambda^{2}\right)}{\lambda \sqrt{1-\lambda^{2}}} \cdot 2 \lambda K^{3} \leqq(1-\kappa) K
$$

under the assumption $0<\lambda<1$ ?"

The answer is affirmative when the inequality

$$
K=\sqrt{\frac{\sqrt{1-\lambda^{2}}}{52\left(2-\lambda^{2}\right)}} .
$$

holds, because then we have

$$
\left\{\begin{array}{l}
\frac{2\left(2-\lambda^{2}\right)}{\lambda \sqrt{1-\lambda^{2}}} \cdot 24 \lambda K^{2}<\frac{48}{52} \\
\frac{2\left(2-\lambda^{2}\right)}{\lambda \sqrt{1-\lambda^{2}}} \cdot 2 \lambda K^{2}<\frac{4}{52}
\end{array}\right.
$$

so that we can choose such a nonnegative number $\kappa<1$ that the both of inequalities (4.15) and (4.16) may hold.

Summing up the considerations, we have the following

Proposition 4. If $0<\lambda<1$ holds, and if the constant

$$
K=\max \left(\frac{|a|}{\left|1-\nu_{1}{ }^{2}\right|}+\frac{|b|}{\left|1-\nu_{2}{ }^{2}\right|}, \frac{|a| \nu_{1}}{\left|1-\nu_{1}{ }^{2}\right|}+\frac{|b| \nu_{2}}{\left|1-\nu_{2}^{2}\right|}\right)
$$

satisfies the inequality

$$
K<\sqrt{\frac{\sqrt{1-\lambda^{2}}}{52\left(2-\lambda^{2}\right)}}
$$

then the equation (4.1) possesses a quasiperiodic solution $\boldsymbol{x}=\widehat{\boldsymbol{x}}(t)$ with periods $\omega_{1}$ and $\omega_{2}$ such that

$$
\left\|\hat{\boldsymbol{x}}(t)-\boldsymbol{w}_{0}(t ; \lambda)\right\|<K
$$

for all $t$.

Of course the above assumption is a sufficient one for the existence.

\section{3. Galerkin Scheme and an Iterative Process}

Galerkin scheme and an iterative process for the numerical solution of (4.1) are similar to those of the case of Duffing type. So, we shall state the results without explanations, but the notations are the same as 
those in the preceding sections.

The approximate quasiperiodic solution is written in the form

$$
\left\{\begin{array}{l}
x_{m}(t)=\alpha(0,0)+\sum_{r=1}^{m} \sum_{|p|=r}\left\{\alpha_{p} \cos (p, \nu) t+\beta_{p} \sin (p, \nu) t\right\} \\
y_{m}(t)=\frac{d}{d x} x_{m}(t) .
\end{array}\right.
$$

Then we get the following determining equations:

$(4.21)_{1} f_{(0,0)}^{(m)}\left(\alpha^{(m)}\right) \equiv \alpha(0,0)+\lambda \mathcal{G}\left\{x_{m}{ }^{2}(\boldsymbol{u}) y_{m}(\boldsymbol{u})\right\}=0 ，$

$(4.21)_{2} \quad f_{(1,0)}^{(m)}\left(\alpha^{(m)}\right) \equiv\left(1-\nu_{1}^{2}\right) \alpha(1,0)-2 \lambda \nu_{1} \beta(1,0)-\frac{a}{2}$

$$
+2 \lambda \mathcal{G}\left\{x_{m}{ }^{2}(\boldsymbol{u}) y_{m}(\boldsymbol{u}) \cos \nu_{1} u_{1}\right\}=0,
$$

$(4.21)_{3} \quad f_{(0,1)}^{(m)}\left(\alpha^{(m)}\right) \equiv\left(1-\nu_{2}^{2}\right) \alpha(0,1)-2 \lambda \nu_{2} \beta(0,1)-\frac{b}{2}$

$$
+2 \lambda \mathcal{G}\left\{x_{m}{ }^{2}(\boldsymbol{u}) y_{m}(\boldsymbol{u}) \cos \nu_{2} u_{2}\right\}=0,
$$

(4. 21) ${ }_{4} \quad f_{\left(p_{1}, p_{2}\right)}^{(m)}\left(\alpha^{(m)}\right) \equiv\left\{1-\left(p_{1} \nu_{1}+p_{2} \nu_{2}\right)^{2}\right\} \alpha_{p}-2 \lambda\left(p_{1} \nu_{1}+p_{2} \nu_{2}\right) \beta_{p}$

$$
+2 \lambda \mathcal{G}\left\{x_{m}{ }^{2}(\boldsymbol{u}) y_{m}(\boldsymbol{u}) \cos (p, \nu, \boldsymbol{u})\right\}=0 \text { for }|p| \geqq 2 \text {, }
$$

(4. 21) $)_{5} \quad g_{\left(p_{1}, p_{2}\right)}^{(m)}\left(\alpha^{(m)}\right) \equiv\left\{1-\left(p_{1} \nu_{1}+p_{2} \nu_{2}\right)^{2}\right\} \beta_{p}+2 \lambda\left(p_{1} \nu_{1}+p_{2} \nu_{2}\right) \alpha_{p}$

$$
+2 \lambda \mathcal{G}\left\{x_{m}{ }^{2}(u) y_{m}(u) \sin (p, \nu, u)\right\}=0 \text { for }|p| \geqq 1 \text {. }
$$

For brevity, let us denote the determing equations $(4.21)_{1} \sim(4.21)_{5}$ by $G_{m}\left(\alpha^{(m)}\right)=0$.

The iterative process:

Starting from a suitable initial value $\alpha_{0}{ }^{(m)}$, we make a sequence of vectors $\left\{\alpha_{k}{ }^{(m)}\right\}$ such that

$$
\alpha_{k}{ }^{(m)}=-\lambda B_{m}{ }^{-1} D_{m}\left(\alpha_{k-1}^{(m)}\right)+B_{m}{ }^{-1} \boldsymbol{a}, k=1,2, \cdots,
$$

where

$$
B_{m}=\left(\begin{array}{cccc}
1 & & \\
1-\nu_{1}{ }^{2} & -2 \lambda \nu_{1} & & \\
2 \lambda \nu_{1} & 1-\nu_{1}{ }^{2} & & \\
& 1-\nu_{2}{ }^{2} & -2 \lambda \nu_{2} & \\
& 2 \lambda \nu_{2} & 1-\nu_{2}^{2} & \\
& & i-\left(p_{1} \nu_{1}+p_{2} \nu_{2}\right)^{2} & -2 \lambda\left(p_{1} \nu_{1}+p_{2} \nu_{2}\right) \\
& & 2 \lambda\left(p_{1} \nu_{1}+p_{2} \nu_{2}\right) & 1-\left(p_{1} \nu_{1}+p_{2} \nu_{2}\right)^{2} \\
& & & \ddots
\end{array}\right),
$$


(4. 24) $\boldsymbol{a}={ }^{t}(0, a / 2,0, b / 2,0, \cdots, 0)$, and $D_{m}\left(\alpha^{(m)}\right)$ is the nonlinear function of $\alpha^{(m)}$

$$
D_{m}\left(\alpha^{(m)}\right)={ }^{t}\left(\zeta_{0}, \cdots, \zeta_{p}, \eta_{p}, \cdots\right)_{|p| \leqq m, p_{1} \geqq 0},
$$

where

$$
\left\{\begin{array}{l}
\zeta_{p}=2 \mathcal{G}\left\{x_{m}{ }^{2}(\boldsymbol{u}) y_{m}(\boldsymbol{u}) \cos (p, \boldsymbol{\nu}, \boldsymbol{u})\right\} \\
\eta_{p}=2 \mathcal{G}\left\{x_{m}{ }^{2}(\boldsymbol{u}) y_{m}(\boldsymbol{u}) \sin (p, \nu, \boldsymbol{u})\right\} \\
\zeta_{\boldsymbol{0}}=\mathcal{G}\left\{x_{m}{ }^{2}(\boldsymbol{u}) y_{m}(\boldsymbol{u})\right\}
\end{array}\right.
$$

4. 4. A posteriori Estimates

After some itertions, we have an approximate solution $\bar{\alpha}^{(m)}$ of the determining equation. The vector $\bar{\alpha}^{(m)}$ gives an approximate solution $\bar{x}_{m}(\mathrm{t})$ such that

(4. 27) $\quad \bar{x}_{m}(t)=\bar{\alpha}(0,0)+\sum_{r=1}^{m} \sum_{|p|=r}\left\{\bar{\alpha}_{p} \cos (p, \nu) t+\bar{\beta}_{p} \sin (p, \nu) t\right\}$.

The residual function to $\bar{x}_{m}(t)$ is as follows:

$$
\begin{aligned}
r(t)= & \frac{d^{2} \bar{x}_{m}(t)}{d t^{2}}-2 \lambda\left(1-\bar{x}_{m}{ }^{2}(t)\right) \frac{d \bar{x}_{m}(t)}{d t} \\
& +\bar{x}_{m}(t)-a \cos \nu_{1} t-b \cos \nu_{2} t .
\end{aligned}
$$

The residual function $r(t)$ is expanded into the finite double Fourier series as follows:

$$
r(t)=f_{(0,0)}+\sum_{r=1}^{3 m} \sum_{i p \mid=r}\left\{f_{p} \cos (p, \nu) t+g_{p} \sin (p, \nu) t\right\},
$$

where

$$
\left\{\begin{array}{l}
f_{(0,0)}=f_{(0,0)}^{(m)}\left(\bar{\alpha}^{(m)}\right), \\
f_{p}= \begin{cases}f_{p}^{(m)}\left(\bar{\alpha}^{(m)}\right) & \text { for }|p| \leqq m, \\
2 \lambda \mathcal{G}\left\{\bar{x}_{m}{ }^{2}(\boldsymbol{u}) \bar{y}_{m}(\boldsymbol{u}) \cos (p, \nu, \boldsymbol{u})\right\} & \text { for }|p|>m,\end{cases} \\
g_{p}= \begin{cases}g_{p}^{(m)}\left(\bar{\alpha}^{(m)}\right) & \text { for }|p| \leqq m, \\
2 \lambda \mathcal{G}\left\{\bar{x}_{m}^{2}(\boldsymbol{u}) \bar{y}_{m}(\boldsymbol{u}) \sin (p, \boldsymbol{\nu}, \boldsymbol{u})\right\} & \text { for }|p|>m .\end{cases}
\end{array}\right.
$$

Let us set 


$$
r=\left|f_{(0,0)}\right|+\sum_{r=1}^{3 m} \sum_{|p|=r}\left\{\left|f_{p}\right|+\left|g_{p}\right|\right\},
$$

then we have

$$
|r(t)| \leqq r \text { for all } t
$$

Define

$$
\Omega=\left|\bar{\alpha}_{(0,0)}\right|+\sum_{r=1}^{m} \sum_{|p|=r}\left(\left|\bar{\alpha}_{p}\right|+\left|\bar{\beta}_{p}\right|\right),
$$

and

$$
\Omega^{\prime}=\sum_{r=1}^{m} \sum_{|p|=r}|(p, \nu)|\left(\left|\bar{\alpha}_{p}\right|+\left|\bar{\beta}_{p}\right|\right),
$$

then we have

$$
\Omega \geqq \sup \left|\bar{x}_{m}(t)\right|, \quad \Omega^{\prime} \geqq \sup \left|\bar{y}_{m}(t)\right| .
$$

For $x$ which is in the $\delta$-neighbourhood of $\bar{x}_{m}(t)={ }^{t}\left(\bar{x}_{m}(t), \bar{y}_{m}(t)\right)$, we have

(4. 36) $\|\Psi(x ; \lambda)-A(\lambda)\| \leqq 2 \lambda\left\{\Omega\left(2 \Omega^{\prime}+\Omega\right)+2\left(\Omega^{\prime}+2 \Omega\right) \delta+3 \delta^{2}\right\}$.

By (4.14), for the Green function $G_{\lambda}(t, s)$ we have

$$
M=\frac{2\left(2-\lambda^{2}\right)}{\lambda \sqrt{1-\lambda^{2}}} .
$$

If there exist a non-negative number $\kappa<1$ and a positive number $\hat{o}$ satisfying the both of inequalities

$$
\begin{gathered}
2 \lambda\left\{\Omega\left\{2 \Omega^{\prime}+\Omega\right)+2\left(\Omega^{\prime}+2 \Omega\right) \delta+3 \delta^{2}\right\} \leqq \frac{\lambda \sqrt{1-\lambda^{2}}}{2\left(2-\lambda^{2}\right)} \kappa \\
\frac{r}{1-\kappa} \cdot \frac{2\left(2-\lambda^{2}\right)}{\lambda \sqrt{ } 1-\lambda^{2}} \leqq \delta
\end{gathered}
$$

then by the Theorem the exact quasiperiodic solution $\widehat{x}(t)$ with periods $\omega_{1}$ and $\omega_{2}$ exists and an error estimation of $\overline{\boldsymbol{x}}_{m}(t)$ is given by

$$
\left\|\bar{x}_{m}(t)-\widehat{x}(t)\right\| \leqq \frac{r}{1-\kappa} \cdot \frac{2\left(2-\lambda^{2}\right)}{\lambda \sqrt{1-\lambda^{2}}},
$$

i.e.

$$
\text { (4. 41) }\left|\overline{\boldsymbol{x}}_{m}(t)-\hat{\boldsymbol{x}}(t)\right|,\left|\frac{d}{d t} \overline{\boldsymbol{x}}_{m}(t)-\frac{d}{d t} \hat{\boldsymbol{x}}(t)\right| \leqq \frac{r}{1-\kappa} \cdot \frac{2\left(2-\lambda^{2}\right)}{\lambda \sqrt{1-\lambda^{2}}}
$$


for all $t$.

\section{§5. Numerical Results}

In the present section we shall show some numerical results obtained by digital computers. The computations have been carried out by TOSBAC 3400 in the Research Institute for Mathematical Sciences and FACOM 230-60 in the Data Processing Center, Kyoto University.

The notations are the same as those in the preceding sections.

\section{1. Numerical Techniques}

In Duffing type case, as the initial value $\alpha_{0}^{(m)}$ for the iteration, we use the values when $\varepsilon=0$, that is, from (3.6)

$$
\left\{\begin{array}{l}
\alpha_{0}(1,0)=\frac{1}{2} \frac{\left(\nu^{2}-\nu_{1}^{2}\right) a}{\left(\nu^{2}-\nu_{1}^{2}\right)^{2}+4 \sigma^{2} \nu_{1}^{2}}, \\
\beta_{0}(1,0)=\frac{\sigma \nu_{1} a}{\left(\nu^{2}-\nu_{1}^{2}\right)^{2}+4 \sigma^{2} \nu_{1}^{2}}, \\
\alpha_{0}(0,1)=\frac{1}{2} \frac{\left(\nu^{2}-\nu_{2}^{2}\right) b}{\left(\nu^{2}-\nu_{2}^{2}\right)^{2}+4 \sigma^{2} \nu_{2}^{2}}, \\
\beta_{0}(0,1)=\frac{\sigma \nu_{2} b}{\left(\nu^{2}-\nu_{2}^{2}\right)^{2}+4 \sigma^{2} \nu_{2}^{2}},
\end{array}\right.
$$

and others equal to 0 .

When we compute the values of $C_{m}\left(\alpha^{(m)}\right)$ for the known coefficients $\alpha^{(m)}$, we adopt FFT method twice for the variables $u_{1}$ and $u_{2}$. Given a suitable small positive number $\Delta$ and a positive integer $I$ (maximum number of iterations), if, convergence criterion

$$
\left\|\alpha_{k}^{(m)}-\alpha_{k-1}^{(m)}\right\| \leqq \Delta \text { for some } k<I
$$

holds, then we regard that the sequence $\left\{\alpha_{k}{ }^{(m)}\right\}$ has converged and stop the iterative computation.

In the case of van der Pol type, the numerical techniques are similar to the above.

\section{2. Duffing type equation}

The constants of system are put as $\sigma=\frac{1}{8}, \nu=\sqrt{2}$. Then, we have 


$$
0.025787023<\frac{\sigma \sqrt{\nu^{2}-\sigma^{2}}}{2 \nu(1+\nu)}<0.025787024
$$

The frequencies of the forcing term are $\nu_{1}=1, \nu_{2}=\sqrt{ } \overline{5}$.

Example 1. The case $\varepsilon=\frac{1}{32}, a=\frac{1}{8}, b=\frac{1}{2} . \quad m=8, \quad \Delta=10^{-8} .3$ iterations.

(5. 4) $\quad \bar{x}_{8}(t)=2\left\{0.0589905 \cos \nu_{1} t+0.0147061 \sin \nu_{1} t\right.$

$-0.0804708 \cos \nu_{2} t+0.0150072 \sin \nu_{2} t$

$-0.0000016 \cos 3 \nu_{1} t+0.0000015 \sin 3 \nu_{1} t$

$+0.0000069 \cos \left(2 \nu_{1}+\nu_{2}\right) t-0.0000026 \sin \left(2 \nu_{1}+\nu_{2}\right) t$

$-0.0000468 \cos \left(2 \nu_{1}-\nu_{2}\right) t+0.0000376 \sin \left(2 \nu_{1}-\nu_{2}\right) t$

$-0.0000054 \cos \left(\nu_{1}+2 \nu_{2}\right) t-0.0000004 \sin \left(\nu_{1}+2 \nu_{2}\right) t$

$-0.0000116 \cos \left(\nu_{1}-2 \nu_{2}\right) t+0.0000076 \sin \left(\nu_{1}-2 \nu_{2}\right) t$

$+0.0000007 \cos 3 \nu_{2} t+0.0000004 \sin 3 \nu_{2} t$

$+0.0000001 \cos \left(4 \nu_{1}-\nu_{2}\right) t$

$\left.-0.0000003 \cos \left(3 \nu_{1}-2 \nu_{2}\right) t-0.0000005 \sin \left(3 \nu_{1}-2 \nu_{2}\right) t\right\}$

(All the terms whose coefficients are smaller than $10^{-7}$ in magnitude are omitted.)

By (3. 27) and (3. 28), we can take $r=1.4 \times 10^{-9}$ and $\omega=0.3385969$.

If we take $\delta=0.125$ and $\kappa=0.78136$, the following inequalities hold:

$$
\begin{aligned}
& 3 \varepsilon(\omega+\delta)^{2} \leqq 0.02014895 \leqq \frac{\kappa \sigma \sqrt{\nu^{2}-\sigma^{2}}}{2 \nu(1+\nu)}, \\
& \frac{r}{1-\kappa} \cdot \frac{2 \nu(1+\nu)}{\sigma \sqrt{\nu^{2}-\sigma^{2}}} \leqq 2.5 \times 10^{-7}<\delta
\end{aligned}
$$

Hence the approximate solution $\bar{x}_{8}(t)$ of (5.4) satisfies the both inequalities (3.34) and (3.35), and we can assume that the exact quasiperiodic solution $\hat{x}(t)$ exists satisfying

$$
\left|\bar{x}_{8}(t)-\widehat{x}(t)\right| \leqq 2.5 \times 10^{-7}
$$

for all $t$.

The values of $\delta$ and $\kappa$ which are chosen to satisfy the both inequal- 
ities (3.34) and (3.35), and other data for Examples $2 \sim 5$ are shown in Table 1.

Table 1.

\begin{tabular}{c||c|c|c|c|c|c|c|c|c||c|c|c}
\hline Ex. & $\varepsilon$ & $a$ & $b$ & $m$ & $\begin{array}{c}\text { Number of } \\
\text { iterations }\end{array}$ & $\omega$ & $n$ & $r$ & $\delta$ & $\kappa$ & $\begin{array}{c}\text { error } \\
\text { bound }\end{array}$ \\
\hline \multirow{2}{*}{1} & $\frac{1}{32}$ & $\frac{1}{8}$ & $\frac{1}{2}$ & 8 & 3 & 0.3385969 & 24 & $1.4 \times 10^{-9}$ & $\frac{1}{8}$ & 0.78136 & $2.5 \times 10^{-7}$ \\
2 & $\frac{1}{64}$ & $\frac{1}{4}$ & $\frac{1}{2}$ & 8 & 4 & 0.4860409 & 24 & $1.5 \times 10^{-9}$ & $\frac{1}{8}$ & 0.6788 & $1.9 \times 10^{-7}$ \\
3 & $\frac{1}{32}$ & $\frac{1}{4}$ & $\frac{1}{2}$ & 8 & 4 & 0.4868917 & 24 & $4.2 \times 10^{-9}$ & $\frac{1}{32}$ & 0.97604 & $6.8 \times 10^{-6}$ \\
4 & $\frac{1}{16}$ & $\frac{1}{8}$ & $\frac{1}{2}$ & 8 & 4 & 0.3390548 & 24 & $2.8 \times 10^{-8}$ & $\frac{3}{128}$ & 0.95543 & $2.5 \times 10^{-8}$ \\
5 & $\frac{1}{8}$ & $\frac{1}{8}$ & $\frac{1}{4}$ & 8 & 4 & 0.2434459 & 24 & $2.1 \times 10^{-8}$ & $\frac{1}{64}$ & 0.97604 & $4.1 \times 10^{-6}$ \\
\hline
\end{tabular}

Example 2. $\varepsilon=\frac{1}{64}, a=\frac{1}{4}, b=\frac{1}{2}, \Delta=10^{-8}$.

$$
\begin{aligned}
\bar{x}_{8}(t) & =2\left\{0.1179220 \cos \nu_{1} t+0.0294121 \sin \nu_{1} t\right. \\
& -0.0804519 \cos \nu_{2} t+0.0150071 \sin \nu_{2} t \\
& -0.0000065 \cos 3 \nu_{1} t+0.0000059 \sin 3 \nu_{1} t \\
& +0.0000138 \cos \left(2 \nu_{1}+\nu_{2}\right) t-0.0000051 \sin \left(3 \nu_{1}+\nu_{2}\right) t \\
& -0.0000936 \cos \left(2 \nu_{1}+\nu_{2}\right) t+0.0000751 \sin \left(2 \nu_{1}-\nu_{2}\right) t \\
& -0.0000054 \cos \left(\nu_{1}+2 \nu_{2}\right) t-0.0000004 \sin \left(\nu_{1}+2 \nu_{2}\right) t \\
& -0.0000116 \cos \left(\nu_{1}-2 \nu_{2}\right) t+0.0000076 \sin \left(\nu_{1}-2 \nu_{2}\right) t \\
& +0.0000003 \cos 3 \nu_{2} t+0.0000002 \sin 3 \nu_{2} t \\
& +0.0000002 \cos \left(4 \nu_{1}-\nu_{2}\right) t \\
& \left.-0.0000006 \cos \left(3 \nu_{1}-2 \nu_{2}\right) t-0.0000010 \sin \left(3 \nu_{1}-2 \nu_{2}\right) t\right\} .
\end{aligned}
$$

Example 3. $\varepsilon=\frac{1}{32}, a=\frac{1}{4}, b=\frac{1}{2}, \Delta=10^{-8}$.

$$
\begin{aligned}
\bar{x}_{8}(t) & =2\left\{0.1181992 \cos \nu_{1} t+0.0294132 \sin \nu_{1} t\right. \\
& -0.0803665 \cos \nu_{2} t+0.0150070 \sin \nu_{2} t \\
& -0.0000131 \cos 3 \nu_{1} t+0.0000119 \sin 3 \nu_{1} t \\
& +0.0000277 \cos \left(2 \nu_{1}+\nu_{2}\right) t-0.0000103 \sin \left(2 \nu_{1}+\nu_{2}\right) t
\end{aligned}
$$


Numerical QUasiperiodic DifFERENTIAL EQuations

$-0.0001886 \cos \left(2 \nu_{1}-\nu_{2}\right) t+0.0001500 \sin \left(2 \nu_{1}-\nu_{2}\right) t$

$-0.0000107 \cos \left(\nu_{1}+2 \nu_{2}\right) t-0.0000008 \sin \left(\nu_{1}+2 \nu_{2}\right) t$

$-0.0000232 \cos \left(\nu_{1}-2 \nu_{2}\right) t+0.0000151 \sin \left(\nu_{1}-2 \nu_{2}\right) t$

$+0.0000007 \cos 3 \nu_{2} t+0.0000004 \sin 3 \nu_{2} t$

$+0.0000008 \cos \left(4 \nu_{1}-\nu_{2}\right) t-0.0000002 \sin \left(4 \nu_{1}-\nu_{2}\right) t$

$\left.-0.0000026 \cos \left(3 \nu_{1}-2 \nu_{2}\right) t-0.0000039 \sin \left(3 \nu_{1}-2 \nu_{2}\right) t\right\}$.

Example 4. $s=\frac{1}{16}, a=\frac{1}{8}, b=\frac{1}{2}, \Delta=10^{-3}$.

$$
\begin{aligned}
\bar{x}_{8}(t) & =2\left\{0.0591585 \cos \nu_{1} t+0.0147067 \sin \nu_{1} t\right. \\
& -0.0804045 \cos \nu_{2} t+0.0150072 \sin \nu_{2} t \\
& -0.0000033 \cos 3 \nu_{1} t+0.0000030 \sin 3 \nu_{1} t \\
& +0.0000139 \cos \left(2 \nu_{1}+\nu_{2}\right) t-0.0000051 \sin \left(2 \nu_{1}+\nu_{2}\right) t \\
& -0.0000945 \cos \left(2 \nu_{1}-\nu_{2}\right) t+0.0000752 \sin \left(2 \nu_{1}-\nu_{2}\right) t \\
& -0.0000108 \cos \left(\nu_{1}+2 \nu_{2}\right) t-0.0000008 \sin \left(\nu_{1}+2 \nu_{2}\right) t \\
& -0.0000232 \cos \left(\nu_{1}-2 \nu_{2}\right) t+0.0000152 \sin \left(\nu_{1}-2 \nu_{2}\right) t \\
& +0.0000013 \cos 3 \nu_{2} t+0.0000008 \sin 3 \nu_{2} t \\
& +0.0000002 \cos \left(4 \nu_{1}-\nu_{2}\right) t \\
& \left.-0.0000013 \cos \left(3 \nu_{1}-2 \nu_{2}\right) t-0.0000019 \sin \left(3 \nu_{1}-2 \nu_{2}\right) t\right\}
\end{aligned}
$$

Example 5. $\varepsilon=\frac{1}{8}, a=\frac{1}{8}, b=\frac{1}{4}, \Delta=10^{-8}$.

$$
\begin{aligned}
\bar{x}_{8}(t) & =2\left\{0.0590996 \cos \nu_{1} t+0.0147066 \sin \nu_{1} t\right. \\
& -0.0401832 \cos \nu_{2} t+0.0075035 \sin \nu_{2} t \\
& -0.0000065 \cos 3 \nu_{1} t+0.0000059 \sin 3 \nu_{1} t \\
& +0.0000138 \cos \left(2 \nu_{1}+\nu_{2}\right) t-0.0000051 \sin \left(2 \nu_{1}+\nu_{2}\right) t \\
& -0.0000943 \cos \left(2 \nu_{1}-\nu_{2}\right) t+0.0000750 \sin \left(2 \nu_{1}-\nu_{2}\right) t \\
& -0.0000054 \cos \left(\nu_{1}+2 \nu_{2}\right) t+0.0000004 \sin \left(\nu_{1}+2 \nu_{2}\right) t \\
& -0.0000116 \cos \left(\nu_{1}-2 \nu_{2}\right) t+0.0000076 \sin \left(\nu_{1}-2 \nu_{2}\right) t
\end{aligned}
$$


$+0.0000003 \cos 3 \nu_{2} t+0.0000002 \sin 3 \nu_{2} t$

$+0.0000004 \cos \left(4 \nu_{1}-\nu_{2}\right) t$

$\left.-0.0000013 \cos \left(3 \nu_{1}-2 \nu_{2}\right) t-0.0000019 \sin \left(3 \nu_{1}-2 \nu_{2}\right) t\right\}$

5. 3. Van der Pol type equation

The frequencies of the forcing term are $\nu_{1}=\sqrt{2}, \nu_{2}=\sqrt{ } 5$. iterations.

Example 6. The case $\lambda=\frac{1}{8}, a=\frac{1}{16}, b=\frac{1}{16} . \quad m=8, \Delta=10^{-8} .3$ (5. 5) $\quad \bar{x}_{8}(t)=2\left\{-0.0277900 \cos \nu_{1} t-0.0098404 \sin \nu_{1} t\right.$

$-0.0076639 \cos \nu_{2} t-0.0010748 \sin \nu_{2} t$

$-0.0000009 \cos 3 \nu_{1} t-0.0000006 \sin 3 \nu_{1} t$

$-0.0000011 \cos \left(2 \nu_{1}+\nu_{2}\right) t-0.0000010 \sin \left(2 \nu_{1}+\nu_{2}\right) t$

$+0.0000019 \cos \left(2 \nu_{1}-\nu_{2}\right) t+0.0000043 \sin \left(2 \nu_{1}-\nu_{2}\right) t$

$-0.0000002 \cos \left(\nu_{1}+2 \nu_{2}\right) t-0.0000003 \sin \left(\nu_{1}+2 \nu_{2}\right) t$ $\left.+0.0000006 \sin \left(\nu_{1}-2 \nu_{2}\right) t\right\}$

(Similarly as in 5.1, all the terms whose coefficients are smaller than $10^{-7}$ in magnitude are omitted.)

We can estimate the value $M$ in (4.38) as

$$
32.0009<M=\frac{2\left(2-\lambda^{2}\right)}{\lambda \sqrt{1-\lambda^{2}}}<32.0011 .
$$

By (4. 31), (4. 33) and (4. 34) we can take $r=1.4 \times 10^{-10}$, $\Omega=0.09275984$ and $\Omega^{\prime}=0.1455660$, respectively.

If we take $\delta=0.0625$ and $\kappa=0.7098$, the following inequalities hold:

$$
\begin{aligned}
& 2 \lambda\left(2 \Omega^{\prime}+\Omega+3 \delta\right)(\Omega+\delta) \leqq 0.0110893 \leqq \frac{\lambda \sqrt{1-\lambda^{2}}}{2\left(2-\lambda^{2}\right)} \kappa, \\
& \frac{r}{1-\kappa} \cdot \frac{2\left(2-\lambda^{2}\right)}{\lambda \sqrt{1-\lambda^{2}}}<1.6 \times 10^{-8}<\delta .
\end{aligned}
$$

Hence the approximate solution $\bar{x}_{8}(t)$ of (5.3) satisfies the both inequalities (4.39) and (4.40). Hence we can assure that the exact quasiperiodic solution $\widehat{x}(t)$ exists satisfying 


$$
\left|\bar{x}_{8}(t)-\widehat{x}(t)\right|<1.6 \times 10^{-8}
$$

for all $t$.

For Examples $7 \sim 9$, the values of $\delta$ and $\kappa$, and other data are shown in Table 2.

Table 2 .

\begin{tabular}{c||c|c|c|c|c|c|c|c|c||c|c|c}
\hline Ex. & $\lambda$ & $a$ & $b$ & $m$ & $\begin{array}{c}\text { Number of } \\
\text { iterations }\end{array}$ & $\Omega$ & $\Omega^{\prime}$ & $n$ & $r$ & $\delta$ & $\kappa$ & $\begin{array}{c}\text { error } \\
\text { bound }\end{array}$ \\
\hline 6 & $\frac{1}{8}$ & $\frac{1}{16}$ & $\frac{1}{16}$ & 8 & 3 & 0.09275984 & 0.1455660 & 24 & $1.4 \times 10^{-10}$ & $\frac{1}{16}$ & 0.7098 & $1.6 \times 10^{-8}$ \\
7 & $\frac{1}{16}$ & $\frac{1}{16}$ & $\frac{1}{8}$ & 8 & 3 & 0.1046678 & 0.1753960 & 24 & $1.5 \times 10^{-10}$ & $\frac{1}{16}$ & 0.8599 & $6.9 \times 10^{-8}$ \\
8 & $\frac{1}{4}$ & $\frac{1}{16}$ & $\frac{1}{16}$ & 8 & 3 & 0.08980023 & 0.1422774 & 24 & $2.0 \times 10^{-10}$ & $\frac{3}{32}$ & 0.9933 & $8.8 \times 10^{-8}$ \\
9 & $\frac{1}{2}$ & $\frac{1}{16}$ & $\frac{1}{16}$ & 8 & 3 & 0.06894513 & 0.1127896 & 24 & $1.8 \times 10^{-10}$ & $\frac{3}{32}$ & 0.7572 & $6.0 \times 10^{-9}$ \\
\hline
\end{tabular}

Example 7. $\lambda=\frac{1}{16}, a=\frac{1}{16}, b=\frac{1}{8}, \Delta=10^{-8}$.

$$
\begin{aligned}
\bar{x}_{8}(t) & =2\left\{-0.0303083 \cos \nu_{1} t-0.0053722 \sin \nu_{1} t\right. \\
& -0.0155497 \cos \nu_{2} t-0.0010912 \sin \nu_{2} t \\
& -0.0000003 \cos 3 \nu_{1} t-0.0000005 \sin 3 \nu_{1} t \\
& -0.0000007 \cos \left(2 \nu_{1}+\nu_{2}\right) t-0.0000014 \sin \left(2 \nu_{1}+\nu_{2}\right) t \\
& +0.0000011 \cos \left(2 \nu_{1}-\nu_{2}\right) t+0.0000062 \sin \left(2 \nu_{1}-\nu_{2}\right) t \\
& -0.0000002 \cos \left(\nu_{1}+2 \nu_{2}\right) t-0.0000006 \sin \left(\nu_{1}+2 \nu_{2}\right) t \\
& \left.+0.0000014 \sin \left(\nu_{1}-2 \nu_{2}\right) t\right\}
\end{aligned}
$$

Example 8. $\lambda=\frac{1}{4}, a=\frac{1}{16}, b=\frac{1}{16}, \Delta=10^{-8}$.

$$
\begin{aligned}
\bar{x}_{8}(t) & =2\left\{-0.0208546 \cos \nu_{1} t-0.0147540 \sin \nu_{1} t\right. \\
& -0.0072492 \cos \nu_{2} t-0.0020309 \sin \nu_{2} t \\
& -0.0000013 \cos 3 \nu_{1} t-0.0000002 \sin 3 \nu_{1} t \\
& -0.0000020 \cos \left(2 \nu_{1}+\nu_{2}\right) t-0.0000003 \sin \left(2 \nu_{1}+\nu_{2}\right) t \\
& +0.0000041 \cos \left(2 \nu_{1}-\nu_{2}\right) t+0.0000002 \sin \left(2 \nu_{1}-\nu_{2}\right) t \\
& -0.0000005 \cos \left(\nu_{1}+2 \nu_{2}\right) t-0.0000002 \sin \left(\nu_{1}+2 \nu_{2}\right) t
\end{aligned}
$$




$$
\left.-0.0000001 \cos \left(\nu_{1}-2 \nu_{2}\right) t+0.0000010 \sin \left(\nu_{1}-2 \nu_{2}\right) t\right\}
$$

Example 9. $\lambda=\frac{1}{2}, a=\frac{1}{16}, b=\frac{1}{16}, \Delta=10^{-8}$.

$$
\begin{aligned}
\bar{x}_{8}(t)= & 2\left\{-0.0104283 \cos \nu_{1} t-0.0147438 \sin \nu_{1} t\right. \\
- & 0.0059563 \cos \nu_{2} t-0.0033321 \sin \nu_{2} t \\
& -0.0000008 \sin 3 \nu_{1} t \\
- & 0.0000009 \cos \left(2 \nu_{1}+\nu_{2}\right) t+0.0000011 \sin \left(2 \nu_{1}+\nu_{2}\right) t \\
+ & 0.0000037 \cos \left(2 \nu_{1}-\nu_{2}\right) t-0.0000032 \sin \left(2 \nu_{1}-\nu_{2}\right) t \\
- & 0.0000005 \cos \left(\nu_{1}+2 \nu_{2}\right) t+0.0000001 \sin \left(\nu_{1}+2 \nu_{2}\right) t \\
- & \left.0.0000005 \cos \left(\nu_{1}-2 \nu_{2}\right) t+0.0000011 \sin \left(\nu_{1}-2 \nu_{2}\right) t\right\}
\end{aligned}
$$

\section{Acknowledgement}

The author would like to dedicate the present paper to late Professor Minoru Urabe, who suggested the present problem and gave many helpful discussions while in his life. He also wishes to express sincere thanks to Professor Masatake Mori for his valuable advices and permission to use FFT subprogram made by him.

\section{References}

[1] Brommundt, E., Approximate solutions of quasiperiodic differential equations, $J$. Math. Anal. Appl., 30 (1970), 252-263.

[2] Бурд, В. Ш., Колесов, Ю. С. и Красносельский, М.А., Исследование функции Грина дифференциальных операторов с почти периодическими козффициентами, Изв. АН СССР, Сер. мат., 33 (1969), 1080-1119.

[3] Мисак, В.В., Устойчивость почти периодического решения обобщенного уравнения ван дер Поля, Вычислительная и Прикладная Математика, 22 (1974), $110-114$.

[4] Urabe, M., Galerkin's procedure for nonlinear periodic systems, Arch. Rational Mech. Anal., 20 (1965), 120-152.

[5] __ Existence theorems of quasiperiodic solutions to nonlinear differential systems, Funkcial. Ekvac., 15 (1972), 75-100.

[6] — On a modified Galerkin's procedure for nonlinear quasiperiodic differential systems, Actes de la Conference Internationale Équations Différentielles et Fonctionelles Non Linéaires, Brussels-Louvain, 1973, 223-258.

[7] - On the existence of quasiperiodic solutions to nonlinear quasiperiodic differential equations, Nonlinear Vibration Problems, Warsaw, 1974, 85-93. 